\title{
REVERSÃO DOS EFEITOS DA HISTÓRIA DE INCONTROLABILIDADE SOB CONTINGÊNCIAS DE VARIAÇÃO COMPORTAMENTAL
}

\author{
Karina de Guimarães Souto e Motta* \\ Josele Abreu-Rodrigues \\ Elisa Tavares Sanabio-Heck
}

\begin{abstract}
RESUMO. A exposição a eventos incontroláveis produz dificuldade na aprendizagem de novos comportamentos. O presente estudo investigou o papel da instrução e da exposição à controlabilidade na reversão dos efeitos da história de incontrolabilidade. No treino, estudantes universitários foram expostos à controlabilidade (grupo CC) ou à incontrolabilidade (grupos IC, ICi, II, IIi). Na "terapia", os grupos CC, IC e ICi foram expostos à controlabilidade, enquanto os grupos II e IIi continuaram expostos à incontrolabilidade. Os grupos ICi e IIi receberam uma instrução de incontrolabilidade/controlabilidade. No teste, todos os grupos foram expostos a controlabilidade. Os participantes expostos apenas à incontrolabilidade (grupos II e IIi) apresentaram maior persistência do responder do que aqueles expostos à "terapia" (grupos IC e ICi), os quais não diferiram dos participantes expostos apenas à controlabilidade (Grupo CC), a despeito da instrução. O procedimento de "terapia", portanto, foi mais efetivo do que a instrução para reverter os efeitos da história de incontrolabilidade.
\end{abstract}

Palavras-chave: desamparo aprendido, "terapia”, instrução.

\section{REVERSING THE EFFECTS OF A HISTORY OF UNCONTROLLABILITY UNDER BEHAVIOR VARIATION CONTINGENCIES}

\begin{abstract}
Pre-exposure to uncontrollable events interferes with subsequent learning of new behaviors. The present study investigated the role of instructions and of the exposure to controllability in reversing the effects of a history of uncontrollability. During training, college students were exposed to controllability (CC Group) or to uncontrollability (IC, ICi, II and Iii groups). During "therapy", the CC, IC and ICi groups were exposed to controllability while the II and IIi groups remained exposed to uncontrollability. An instruction on uncontrollability/controllability was given to the ICi and IIi groups. During testing, all groups were exposed to controllability. The participants exposed only to uncontrollability (II and IIi groups) showed greater response persistence than those exposed to "therapy" (IC and ICi groups) that did not differ from those exposed only to controllability (CC Group). The "therapy" procedure, then, was more effective than instructions in reversing the effects of a history of uncontrollability.
\end{abstract}

Key words: Learned helplessness, therapy, instruction.

\section{REVERSIÓN DE LOS EFECTOS DE LA HISTORIA DE INCONTROLABILIDAD BAJO CONTINGENCIAS DE VARIACIÓN COMPORTAMENTAL}

\footnotetext{
RESUMEN. La exposición a eventos incontrolables produce dificultad en el aprendizaje de nuevos comportamientos. El presente estudio investigó el rol de la instrucción y de la exposición a la controlabilidad en la reversión de los efectos de la historia de incontrolabilidad. En el entrenamiento, estudiantes universitarios fueron expuestos a la controlabilidad (grupo CC) o a la incontrolabilidad (grupos IC, ICi, II, IIi). En la "terapia", los grupos CC, IC y ICi fueron expuestos a la controlabilidad, mientras que los grupos II y IIi siguieron expuestos a la incontrolabilidad. Los grupos ICi y IIi recibieron una instrucción de incontrolabilidad/controlabilidad. En el test,

* Mestre em Psicologia. Universidade de Brasília/Docente do Instituto de Educação Superior de Brasília.

\# Doutora em Psicologia. Docente da Universidade de Brasília.

II Doutora em Psicologia. Docente da Universidade Católica de Goiás.
} 
todos los grupos fueron expuestos a la controlabilidad. Los participantes expuestos sólo a la incontrolabilidad (grupos II y IIi) presentaron mayor persistencia del responder, comparados a aquellos expuestos a la "terapia" (grupos IC y ICi), los cuales no se distinguieron de los participantes expuestos sólo a la controlabilidad (Grupo $\mathrm{CC}$ ), pese a la instrucción. El procedimiento de "terapia", por lo tanto, fue más efectivo que la instrucción para revertir los efectos de la historia de incontrolabilidad.

Palabras-clave: desamparo aprendido, terapia, instrucción.

Quando um organismo é exposto a eventos incontroláveis, isto é, a eventos que ocorrem independentemente do responder, observa-se um retardo na aprendizagem de novas respostas em situações posteriores envolvendo eventos controláveis. Esse fenômeno, denominado de desamparo aprendido, tem sido sistematicamente demonstrado em várias espécies, tais como, cães (e.g., Seligman \& Maier, 1967), ratos (e.g., Williams \& Maier, 1977), pombos (e.g., Goodkin, 1976), galinhas (e.g., Rodd, Rosellini, Stock, \& Gallup, 1997) e humanos (e.g., Hiroto, 1974; Winefield \& Tiggeman, 1978). Ainda, o desamparo é consistentemente observado quando os eventos incontroláveis são aversivos (e.g., choques elétricos), o que nem sempre ocorre quando eventos apetitivos (e.g., comida, água) são utilizados (Capelari \& Hunziker, 2005; Goodkin, 1976).

O estudo de Seligman e Maier (1967, Experimento 1) ilustra a metodologia-padrão na literatura de desamparo. $\mathrm{Na}$ fase de treino, um grupo de cães foi exposto a choques controláveis, que poderiam ser interrompidos pela emissão da resposta de pressionar um painel (grupo controlabilidade). Um segundo grupo foi acoplado ao primeiro em relação à duração, intensidade e número de choques, porém a interrupção dos choques não era possível (grupo incontrolabilidade). Um terceiro grupo não recebeu choques (grupo controle). $\mathrm{Na}$ fase de teste, todos os sujeitos foram expostos a choques que poderiam ser interrompidos pela emissão de uma nova resposta de fuga (saltar uma barreira). Os resultados mostraram que os sujeitos do grupo incontrolabilidade não aprenderam a resposta de saltar, o que não ocorreu com aqueles dos grupos controlabilidade e controle, os quais apresentaram desempenhos semelhantes.

Diversos pesquisadores têm utilizado o procedimento experimental conhecido como "terapia" para reverter os efeitos da história de exposição a incontrolabilidade (e.g., Klein \& Seligman, 1976; Nation \& Massad, 1978; Seligman, Rosellini \& Kozak, 1975; Williams \&
Maier, 1977). Esses estudos têm indicado que quando sujeitos são expostos a eventos incontroláveis e, em seguida, a eventos controláveis, não ocorre retardo na aprendizagem posterior de uma nova tarefa. Os efeitos do procedimento de "terapia" foram relatados por Williams e Maier (1977, Experimento 2). Na fase de treino desse estudo, dois grupos de ratos (I e II) foram expostos a choques incontroláveis. $\mathrm{Na}$ fase de "terapia", um grupo que havia recebido choque na fase anterior (grupo I) e um que não havia recebido choque (grupo III) foram expostos a choques que poderiam ser interrompidos por meio da resposta de pressão à barra. Dois outros grupos (II e IV) não foram expostos a essa fase. Durante a fase de teste, todos os grupos foram expostos a choques que poderiam ser interrompidos ou evitados por meio da resposta de ir e voltar em uma caixa com dois compartimentos. Os dois grupos que foram inicialmente expostos a choques incontroláveis (I e II) apresentaram dificuldade na aprendizagem do responder durante a fase de teste, o que não foi observado com os grupos que não tiveram essa experiência (III e IV). Dentre os grupos I e II, aquele exposto à fase de "terapia" (Grupo I) aprendeu eventualmente a resposta de fuga durante o teste, o mesmo não ocorrendo com o grupo que não foi exposto a essa fase (Grupo II). Resultados comparáveis também foram observados com humanos (e.g., Klein \& Seligman, 1976; Nation \& Massad, 1978).

Os estudos mostram que o desamparo aprendido é afetado não somente pelo procedimento de "terapia", mas também por diversas outras variáveis, como, por exemplo, o conteúdo das instruções (Hiroto, 1974; Klein, Fencil-Morse \& Seligman, 1976; Mikulincer, 1986; Tennen, Gillen \& Drum, 1982). No estudo de Klein e cols. (1976), estudantes universitários foram expostos a uma tarefa de resolução de problemas de discriminação visual durante a fase de treino. O grupo solucionável recebia feedbacks de acerto e erro consistentes com suas respostas a cada tentativa. Para os grupos insolucionáveis, 
esses feedbacks eram apresentados independentemente das respostas dos participantes. O grupo insolucionável-habilidade recebeu uma instrução informando que, em experimentos prévios, a maioria das pessoas foi capaz de solucionar aquela tarefa, enquanto o grupo insolucionável-sorte recebeu uma instrução indicando que a maioria das pessoas não conseguiu solucionar a tarefa. O grupo controle não foi exposto à tarefa de discriminação. Na fase de teste, todos os participantes tinham que resolver anagramas. O grupo insolucionávelhabilidade apresentou tempos de reação mais longos e um maior número de falhas na tarefa do anagrama do que os grupos insolucionável-sorte, solucionável e controle, os quais não diferiram entre si. Esses resultados sugerem que, dependendo do conteúdo das instruções, a exposição a incontrolabilidade não produz, necessariamente, retardo na aprendizagem de novas respostas.

Em suma, a literatura aponta que o procedimento de "terapia" e o uso de instruções podem atenuar ou mesmo eliminar o desamparo aprendido. Entretanto, uma vez que esses procedimentos foram investigados em estudos com metodologias diferentes, é difícil apontar a contribuição relativa de cada um para a reversão dos efeitos da história de incontrolabilidade. Dessa forma, o objetivo do presente estudo consistiu em comparar, a partir de uma mesma metodologia, a eficácia do procedimento de "terapia" e da apresentação de instruções. Mais especificamente, objetivou-se investigar se a exposição à "terapia" produziria efeitos comparáveis àqueles produzidos por uma instrução que explicitasse a relação de incontrolabilidade presente na fase de treino e a relação de controlabilidade presente na fase de "terapia". Um objetivo adicional consistiu em estender as investigações sobre desamparo aprendido para o contexto de variação comportamental. Para tanto, na fase de teste, o efeito da história de incontrolabilidade foi avaliado em uma situação em que o reforço era contingente a variabilidade.

\section{MÉTODO}

\section{Participantes}

Participaram do experimento 30 alunos do curso de graduação em Psicologia da
Universidade de Brasília, sendo 15 do sexo masculino e 15 do sexo feminino, sem experiência prévia com procedimentos experimentais. Cada participante ganhou 0,5 ponto por cada hora de participação. Os pontos ganhos poderiam ser adicionados ao total de pontos de uma disciplina à sua escolha, desde que o participante já estivesse aprovado nessa disciplina.

\section{Equipamento}

O experimento foi conduzido em uma sala com $1,70 \mathrm{~m} \times 1,30 \mathrm{~m}$, dotada de isolamento acústico. Foi utilizado um microcomputador, modelo Pentium II, para a coleta de dados. O controle das contingências experimentais e o registro dos dados foram realizados por um programa desenvolvido em linguagem Visual Basic $6^{\circledR}$. Nas fases de treino e teste, um tom de $90 \mathrm{~dB}$ com $3.000 \mathrm{~Hz}$, previamente julgado como moderadamente aversivo (Hiroto, 1974; Klein \& Seligman, 1976; Nation \& Massad, 1978), era apresentado por meio de fones de ouvido.

\section{Procedimento}

Os participantes foram divididos semialeatoriamente em seis grupos com cinco participantes cada, os quais foram expostos a três fases experimentais (treino, "terapia" e teste), conforme apresentado na Tabela 1.

Tabela 1. Contingências Programadas nas Fases de Treino, "Terapia" e Teste Para Cada Grupo Experimental.

\begin{tabular}{cccc}
\hline Condições & Treino & "Terapia" & Teste \\
\hline CC & C & C & C \\
IC & I & C & C \\
ICi & I & C + instrução & C \\
II & I & I & C \\
IIi & I & I + instrução & C \\
Ct & - & - & C \\
\hline
\end{tabular}

C: Controlabilidade; I: Incontrolabilidade; i: Instrução; Ct: Controle

\section{Treino}

No início dessa fase, a cada participante era dada a opção de deixar o laboratório após ouvir amostras breves de um tom. Caso o participante optasse por permanecer, iniciava-se a sessão. Cada tentativa era iniciada com a apresentação do tom e finalizada com seu término. Após um período de $1 \mathrm{~s}$ de apresentação do tom, apareciam 
quatro círculos na tela do computador, alinhados horizontalmente e com a mesma cor da tela. O participante, então, deveria emitir uma seqüência de quatro respostas de pressionar as teclas $\mathrm{F}$ e J do teclado. Quando a primeira resposta era emitida, o primeiro círculo, à esquerda, era iluminado de azul (se a resposta fosse na tecla F) ou amarelo (se a resposta fosse na tecla $\mathrm{J}$ ); a segunda resposta iluminava o segundo círculo, e assim sucessivamente. Havia um intervalo entre respostas de $0.5 \mathrm{~s}$, durante o qual as respostas emitidas não tinham conseqüências programadas. Uma seqüência era considerada correta quando continha duas respostas na tecla $\mathrm{F}$ e duas na tecla $\mathrm{J}$, não sendo relevante a ordem de emissão dessas respostas na seqüência. Qualquer outra seqüência que não consistisse em uma seqüência $2 \mathrm{~F} 2 \mathrm{~J}$ era considerada incorreta.

Para os participantes do grupo CC, a emissão de uma seqüência correta era seguida pelo término imediato do tom, pela apresentação de um feedback de acerto e pela adição de dois pontos ao contador; a emissão de uma sequiência incorreta, por sua vez, era seguida pela continuação do tom por mais $2 \mathrm{~s}$, por um feedback de erro e pela subtração de dois pontos. Para os participantes dos grupos IC, ICi, II e IIi, os mesmos eventos seguiam a emissão de uma seqüência, mas eram independentes da acurácia desta; ou seja, a ordem de apresentação do término do tom+feedback de acerto+adição de pontos, assim como da continuação do tom+feedback de erro+subtração de pontos, ao longo das tentativas, era previamente determinada pelo experimentador, sendo essa ordem idêntica para todos os participantes desses grupos. Todos os participantes desses cinco grupos receberam a mesma instrução geral sobre a tarefa, a qual não explicitava a relação de independência entre acurácia e eventos subseqüentes. Os participantes do grupo $\mathrm{Ct}$ foram solicitados a ouvir os tons, mas não tinham que emitir seqüências de respostas.

Para o Grupo CC, essa fase era finalizada quando, após um número mínimo de 25 tentativas, o participante emitisse a seqüência correta em cinco tentativas consecutivas. Quando o critério não fosse atendido em, no máximo, 40 tentativas, o participante era dispensado do experimento. Cada participante dos demais grupos foi acoplado a um participante específico do grupo CC em termos do número de tentativas. Dessa forma, o número médio de tentativas foi igual para todos os grupos experimentais.

\section{"Terapia"}

Nessa fase, a tarefa compreendia a resolução de dois problemas de discriminação. Cada problema consistia na apresentação de blocos de oito figuras formadas pela combinação de três dimensões. No problema 1 foram utilizadas as seguintes dimensões: forma (círculo ou quadrado), espessura da moldura (fina ou grossa) e posição da forma (direita ou esquerda). No problema 2, as dimensões consistiam em número (4 ou 8 ), cor do fundo da tela (amarelo ou rosa) e quantidade de molduras (1 ou 3). Antes da sessão o computador selecionava um estímulo e cabia ao participante discriminar, no decorrer das tentativas, qual o estímulo selecionado. A cada tentativa eram apresentadas uma figura e uma pergunta ("A figura apresentada contém a característica selecionada pelo experimentador?") na tela. Para responder à pergunta, o participante deveria clicar com o mouse na palavra SIM ou NÃO, ambas situadas abaixo da pergunta. Por exemplo, se o computador tivesse selecionado, no problema 1 , o estímulo "quadrado" e aparecesse na tela a figura "quadrado com moldura fina e situado à direita da tela", a resposta correta seria "SIM"; se, por outro lado, a figura apresentada fosse "círculo com moldura fina e situado à direita da tela", a resposta correta seria "NÃO". Após cada resposta era fornecido um feedback informando se a resposta estava correta (ganho de dois pontos) ou incorreta (perda de dois pontos).

Para os grupos CC, IC e ICi, o feedback de acerto sempre seguia respostas corretas e o feedback de erro sempre seguia respostas incorretas. Para os grupos II e IIi, os feedbacks eram independentes da resposta do participante. A ordem e distribuição dos feedbacks, idêntica para todos os participantes desses dois grupos, era predeterminada pelo experimentador, de modo que havia um número igual de feedbacks de acerto e de erro.

Todos os grupos receberam a mesma instrução geral sobre a tarefa de discriminação (com exceção do grupo $\mathrm{Ct}$, o qual foi solicitado apenas a olhar as figuras apresentadas na tela). Adicionalmente, os grupos ICi e IIi receberam uma instrução específica sobre as condições presentes na fase de treino e aquelas que ocorreriam na Fase de "terapia" ("Os pontos ganhos ou perdidos na situação anterior não dependiam de suas respostas. Mas, a partir de agora, ganhar ou perder pontos dependerá de seu desempenho"). 
Todos os participantes foram expostos a um mínimo de dois blocos de oito tentativas cada, sendo cada tentativa correspondente a uma figura específica. Para os grupos CC, IC e ICi, cada problema era encerrado quando o participante apresentava oito respostas corretas consecutivas. Se o participante não atingisse esse critério em, no máximo, três blocos, sua participação era cancelada. $\mathrm{O}$ número de tentativas de cada participante dos grupos II, IIi e Ct foi acoplado ao número de tentativas de um participante específico dos grupos IC, ICi e CC, respectivamente.

\section{Teste}

A tarefa era similar àquela da fase de treino, onde os participantes deviam emitir uma seqüência de quatro respostas pressionando as teclas $\mathrm{F}$ e J, com a diferença de que foi implementada uma contingência de variabilidade (critério Lag 3) para os participantes de todos os grupos. Dessa forma, o término do tom, a apresentação do feedback de acerto e a adição de pontos ao contador eram contingentes à emissão de uma seqüência apenas se, e sempre que, ela diferisse das seqüências emitidas nas três últimas tentativas. Se a seqüência fosse idêntica a qualquer uma das três últimas, o tom continuava, o feedback de erro era apresentado e dois pontos eram subtraídos do contador.

Todos os participantes receberam a mesma instrução geral apresentada na fase de treino, inclusive o grupo Ct. Essa fase era finalizada quando, após 25 tentativas, o participante emitisse a seqüência correta em cinco tentativas consecutivas; caso o critério não fosse atingido em, no máximo, 40 tentativas, o experimento era encerrado.

\section{RESULTADOS}

Uma vez que (1) o objetivo do estudo consistiu em avaliar os efeitos da história de incontrolabilidade em situações com e sem instrução e/ou "terapia", e que (2) efeitos de história são transitórios, não foram incluídos na análise a seguir os dados referentes ao período inicial da fase de treino, por mostrarem menos claramente o controle exercido pelas contingências programadas, e ao período final da fase de teste, por revelarem que o controle da história havia diminuído.

A distribuição da freqüência média das 16 sequiências possíveis na segunda e na primeira metades das fases de treino e teste, respectivamente, é mostrada na Figura 1. Essa medida foi obtida dividindo-se o número de ocorrências de cada sequiência pelo número total de seqüências emitidas em cada metade dessas fases. A freqüência de cada uma das seis sequiências $2 \mathrm{~F} 2 \mathrm{~J}$, bem como a freqüência total de sua ocorrência, são mostradas à esquerda da linha pontilhada, enquanto as demais seqüências são apresentadas à direita dessa linha. $\mathrm{Na}$ segunda metade da fase de treino, todos os grupos tenderam a emitir predominantemente seqüências 2F2J, em detrimento das demais seqüências possíveis. $\mathrm{O}$ grupo $\mathrm{CC}$, exposto à controlabilidade, emitiu essas seqüências em $95 \%$ das tentativas. Para os demais grupos (IC, ICi, II e IIi), todos expostos à incontrolabilidade, o total de ocorrências dessas seqüências variou entre $60 \%$ (grupo IIi) e $81 \%$ (grupo II) de todas as seqüências emitidas. Na primeira metade da fase de teste, houve um decréscimo substancial na frequiência de seqüências $2 \mathrm{~F} 2 \mathrm{~J}$ para os grupos expostos à controlabilidade na fase de "terapia" (CC, IC e ICi). Para os grupos II e IIi, expostos à incontrolabilidade nessa mesma fase, o decréscimo foi menos acentuado, e para o grupo $\mathrm{Ct}$, a freqüência dessas seqüências foi comparável àquela dos grupos CC, IC e ICi. Essa diminuição, entretanto, só foi confirmada estatisticamente para os grupos CC, IC e ICi. Ou seja, o teste t unicaudal indicou que a freqüência de seqüências 2F2J dos grupos CC (t [29] = 0,809; p = 0,03), IC $(\mathrm{t}[29]=0,833 ; \mathrm{p}=0,05)$ e ICi (t [29] $=0,763 ; \mathrm{p}$ $=0,07)$ na segunda metade da fase de treino foi significativamente diferente daquela apresentada por esses mesmos grupos na primeira metade da fase de teste. 

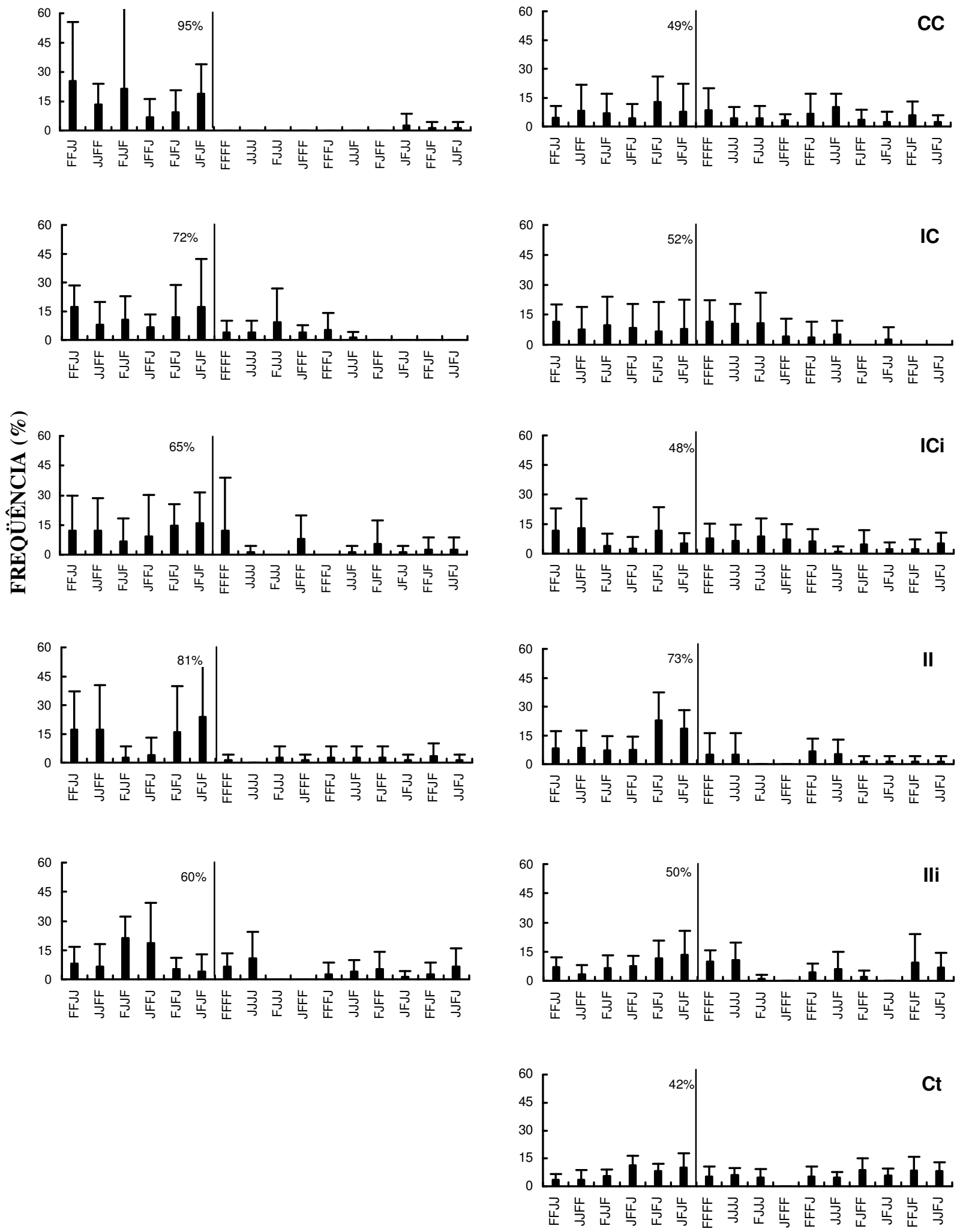

\section{SEQÜÊNCIAS}

Figura 1. Distribuição da Freqüência Média de Cada uma das 16 Sequiências Possíveis na Segunda Metade da Fase de Treino (à Esquerda) e na Primeira Metade da Fase de Teste (à direita) para Todos os Grupos.

À Esquerda da Linha Pontilhada é Apresentada a Freqüência Total das Seqüências 2F2J. As Barras de Erro Representam um Desvio-Padrão 
As diferenças entre as frequiências médias de seqüências $2 \mathrm{~F} 2 \mathrm{~J}$ nas fases de treino e de teste estão resumidas na Figura 2. Essa medida foi obtida subtraindo-se a porcentagem de seqüências $2 \mathrm{~F} 2 \mathrm{~J}$ emitida na primeira metade da fase de teste da porcentagem dessas sequiências na segunda metade da fase de treino. A emissão de seqüências $2 \mathrm{~F} 2 \mathrm{~J}$ diminuiu para todos os grupos (valores abaixo de $0 \%$ ), sendo essa diminuição mais acentuada para o grupo exposto apenas à controlabilidade no decorrer das fases (CC) e menos acentuada para os grupos expostos apenas à incontrolabilidade nas fases de treino e "terapia" (II e IIi). A análise de variância indicou diferenças estatisticamente significativas entre os grupos $(F[4,145]=3,294 ; p=0,013)$. A aplicação do teste Tukey indicou que o grupo CC diferiu dos grupos IC e ICi (que não diferiram entre si) e dos grupos II e IIi (também similares).

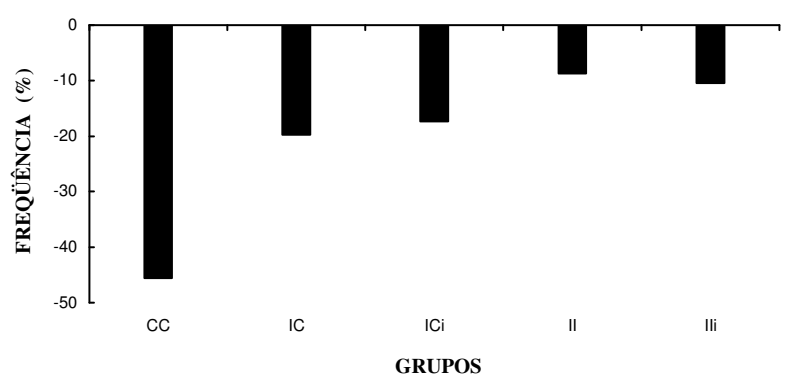

Figura 2. Diferenças entre as freqüências médias das sequiências 2 F2J na segunda metade da fase de treino e na primeira metade da fase de teste para todos os grupos.

Na Fase de "terapia", a maioria dos participantes dos grupos CC, IC e ICi, expostos a eventos controláveis nessa fase, apresentou porcentagens de respostas corretas similares ou superiores a $80 \%$, enquanto para os participantes dos grupos II e IIi, expostos a eventos incontroláveis, a porcentagem foi aproximadamente $50 \%$. A análise de variância apontou diferenças estatisticamente significativas entre os grupos $(F[4,20]=19,942 ; p=0,00)$ e o teste Tukey indicou que essas diferenças ocorreram entre os grupos expostos à controlabilidade e aqueles expostos à incontrolabilidade.

\section{DISCUSSÃO}

O objetivo do presente estudo consistiu em investigar se os efeitos da história de incontrolabilidade seriam revertidos pelo procedimento de "terapia" (grupos IC e ICi), bem como pela apresentação de uma instrução sobre as relações de controlabilidade e de incontrolabilidade presentes nas fases de treino e de "terapia" (grupos ICi e IIi). Os efeitos da história de incontrolabilidade foram avaliados com base na persistência do responder (i.e., da emissão de sequiências 2F2J) quando uma contingência de variação foi implementada na fase de teste. Os resultados mostraram que a "terapia" reduziu os efeitos da história de incontrolabilidade (grupos IC e ICi apresentaram menor persistência que os grupos II e IIi), mas essa redução foi apenas parcial (persistência dos grupos IC e ICi foi maior do que do Grupo CC). Foi também observado que a instrução não minimizou os efeitos da história de incontrolabilidade, ou seja, os grupos com e sem instrução (IC vs. ICi e II vs. IIi) não diferiram em termos da persistência do responder. Os resultados obtidos em todas as fases do experimento serão discutidos a seguir.

\section{Fase de treino}

Nessa fase, a alta incidência de seqüências $2 \mathrm{~F} 2 \mathrm{~J}$ em todos os grupos pode ter resultado de dois fatores: a instrução geral e o reforçamento acidental. A instrução geral informava que deveriam ser emitidas sequiências com quatro respostas de pressionar as teclas $\mathrm{F}$ e J, o que pode ter evocado a distribuição das respostas nessas duas teclas. Para os participantes do grupo CC, essas seqüências foram reforçadas continuamente e, como conseqüência, quase $100 \%$ das seqüências emitidas eram 2F2J. Para os participantes dos grupos expostos à incontrolabilidade, a emissão de sequiências $2 \mathrm{~F} 2 \mathrm{~J}$ produzia eventos reforçadores em $50 \%$ das tentativas, o que promovia a manutenção dessas seqüências. Nas demais tentativas, as sequiências 2F2J geravam eventos aversivos, o que enfraquecia a emissão dessas sequiências e favorecia a emissão de outras seqüências. Assim, a menor incidência de sequiências 2F2J entre os grupos incontroláveis pode refletir a variação comportamental ocasionada pela relação de independência entre o responder e os eventos subseqüentes. Efeitos similares da intermitência do reforço têm sido consistentemente relatados por diversos autores (e.g., Schoenfeld, 1968; Tatham, Wanchisen \& Hineline, 1993).

\section{Fase de "terapia"}

Os participantes dos grupos expostos à controlabilidade nessa fase (CC, IC e ICi) apresentaram um responder discriminado, o mesmo não ocorrendo com aqueles expostos à incontrolabilidade (II e IIi), os quais responderam ao acaso. A instrução que indicava a presença de controlabilidade nessa fase não promoveu uma maior acurácia do responder, já que o grupo controlável com 
instrução (ICi) apresentou resultados semelhantes aos do grupo controlável sem instrução (IC), o mesmo ocorrendo entre os grupos incontroláveis com e sem instrução (IIi e II). É possível que a ausência de controle instrucional tenha ocorrido porque $o$ conteúdo da instrução não especificava o desempenho esperado (Danforth, Chase, Dolan \& Joyce, 1990), mas apenas que ganhar ou perder pontos dependia do desempenho. Além disso, uma vez que havia apenas duas respostas possíveis ("SIM" ou "NÃO") e que cada resposta produzia, necessariamente, uma dentre duas consequiências ("Correto" ou "Incorreto"), a relação de dependência ou independência entre resposta e conseqüência era bastante discriminável, reduzindo, assim, o impacto da instrução. Esse resultado é consistente com aqueles obtidos por Abreu-Rodrigues, Natalino e Aló (2002), Galizio (1979), dentre outros.

\section{Fase de teste}

Para os participantes dos grupos II e IIi, expostos apenas à incontrolabilidade nas fases anteriores, a porcentagem de seqüências 2F2J manteve-se inalterada na primeira metade da fase de teste, a despeito do critério Lag 3 em vigor. Para os demais grupos, foi observada uma diminuição na porcentagem dessas sequiências, principalmente para o grupo exposto apenas à controlabilidade durante $o$ experimento (Grupo CC). Uma vez que os participantes dos grupos II e IIi tenderam a manter o padrão comportamental selecionado na fase de treino e o mesmo não ocorreu com os demais grupos, pode-se afirmar que a história de incontrolabilidade gerou persistência no responder, ou alternativamente, diminuiu a sensibilidade comportamental à implementação do critério de variação. Esses dados corroboram aqueles relatados por estudos sobre o desamparo aprendido (e.g., Hiroto, 1974; Seligman \& Maier, 1967; Winefield \& Tiggemann, 1978).

De acordo com Madden, Chase e Joyce (1998), o comportamento é considerado sensível quando muda diante de mudanças nas contingências. A partir dessa definição, o desempenho do Grupo CC seria sensível ao critério Lag 3 já que houve uma redução substancial na emissão de seqüências 2 F2J. Na fase de treino, esses participantes aprenderam, em função do reforçamento contínuo, que deveriam emitir seqüências 2F2J e que emitir a seqüência reforçada aumentava a probabilidade do reforço. $\mathrm{Na}$ fase de teste, emitir sequiências $2 \mathrm{~F} 2 \mathrm{~J}$ continuava produzindo reforços, uma vez que havia seis sequiências $2 \mathrm{~F} 2 \mathrm{~J}$ e o critério Lag 3 exigia apenas que a seqüência emitida fosse diferente das três anteriores. Porém, repetir a seqüência reforçada não atendia a esse critério e, conseqüentemente, seqüências diferentes de 2F2J passaram a ser emitidas.

Ainda com base na definição de Madden e cols. (1998), o desempenho dos grupos II e IIi deveria ser caracterizado como insensível. Essa afirmativa, no entanto, é questionável. Em decorrência do reforçamento e punição intermitentes durante a fase de treino, os participantes desses grupos desenvolveram um padrão comportamental que incluía a emissão tanto de seqüências $2 \mathrm{~F} 2 \mathrm{~J}$ quanto de outras seqüências. $\mathrm{Na}$ fase de teste, esse padrão atendia ao critério Lag 3, ou seja, era efetivo na produção de reforços e, conseqüentemente, não foi alterado. Assim sendo, a manutenção da porcentagem de sequiências 2F2J não deveria ser caracterizada como insensibilidade, porque não revela ausência de controle pela nova contingência. O mais adequado seria caracterizá-la como pseudo-insensibilidade, conforme proposto por Shimoff, Matthews e Catania (1986).

O desempenho dos grupos IC e ICi foi caracterizado pela redução na ocorrência de sequiências 2F2J, embora essa redução tenha sido menos acentuada do que aquela observada para o grupo CC. Esse resultado sugere que o procedimento de "terapia" atenuou os efeitos da história de incontrolabilidade, conforme apontado por outros estudos sobre o desamparo aprendido (Klein \& Seligman, 1976; Nation \& Massad, 1978; Seligman \& cols., 1975; Williams \& Maier, 1977). Para esse grupo, embora a exposição à incontrolabilidade na fase de treino tenha gerado um responder caracterizado pela emissão de sequiências variadas (assim como ocorreu com os grupos II e IIi), o reforçamento contínuo presente na fase de "terapia" pode ter contribuído para a aprendizagem de que respostas reforçadas deveriam ser repetidas (assim como ocorreu com o grupo $\mathrm{CC}$ ). $\mathrm{Na}$ fase de teste, o padrão aprendido na fase de treino tendeu a ser mantido, porque atendia ao critério Lag 3. Entretanto, uma vez que esse padrão era caracterizado por uma frequiência maior de sequiências $2 \mathrm{~F} 2 \mathrm{~J}$ do que de outras sequiências, houve uma diminuição na frequiência das mesmas porque a repetição da sequiência reforçada gerava eventos punitivos.

$\mathrm{O}$ grupo $\mathrm{Ct}$ apresentou a distribuição mais eqüitativa, dentre todos os grupos, das 16 sequiências possíveis. Provavelmente, esses resultados se devem à ausência de um treino prévio com seqüências com duas respostas na tecla $\mathrm{F}$ e duas respostas na tecla $\mathrm{J}$ e revelam controle pela contingência de variação.

Em suma, o procedimento de "terapia" tem sido amplamente utilizado nos estudos de desamparo 
aprendido com sujeitos não humanos (e.g., Carvalho, 1998; Seligman, Rosellini \& Kozak, 1975; Williams \& Maier, 1977) e humanos (e.g., Klein \& Seligman, 1976; Nation \& Massad, 1978), expostos a esquemas de reforçamento contínuo e intermitente (e.g., Carvalho, 1998; Nation \& Massad, 1978), em ambientes iguais (e.g., Carvalho, 1998; Nation \& Massad, 1978; Seligman, Rosellini \& Kozak, 1975) ou diferentes (e.g., Williams \& Maier, 1977) nas fases de "terapia" e teste. O presente estudo, por sua vez, contribui nas investigações sobre o desamparo aprendido, à medida que mostra a generalização do efeito do procedimento de "terapia" para situações de treino e teste que envolvem não somente um operante complexo, ou seja, a emissão de seqüências de respostas, mas também uma relação resposta-reforço nunca antes utilizada nos estudos de desamparo, a saber, a seleção de variabilidade comportamental.

Além de avaliar os efeitos do procedimento de "terapia", o presente experimento também avaliou os efeitos de uma instrução que indicava que os eventos eram incontroláveis na fase de treino, mas eram controláveis na fase de "terapia". A similaridade entre os desempenhos dos grupos IC e ICi, e dos grupos II e IIi mostra que a apresentação dessa instrução não afetou o desempenho na fase de teste. Essa ausência de controle instrucional é inconsistente com os resultados relatados por Klein e cols. (1976), os quais observaram que uma instrução de sorte, a qual afirmava que a maioria das pessoas não havia conseguido resolver a tarefa, atenuou os efeitos da história de incontrolabilidade. Diferenças no conteúdo das instruções podem ser responsáveis por essa discrepância entre os resultados do presente estudo e os de Klein e cols. (1976). Essa possibilidade parece ser confirmada pelo estudo de Oakes e Curtis (1982). Nesse estudo, metade dos participantes do grupo controlável e metade dos participantes do grupo incontrolável receberam a informação de que os feedbacks tinham sido apresentados independentemente de suas respostas durante a fase de treino. $\mathrm{Na}$ fase de teste, o grupo incontrolável apresentou um maior número de erros ao tentar resolver anagramas do que o grupo controlável, não havendo diferenças entre os participantes que receberam ou não a informação de incontrolabilidade. Esses resultados, em conjunto com os do presente estudo, indicam que a explicitação da relação de incontrolabilidade não é suficiente para eliminar, ou pelo menos atenuar, os efeitos da história de incontrolabilidade.

Algumas características do procedimento aqui utilizado devem ser destacadas. A primeira característica refere-se à programação dos eventos incontroláveis. Na situação de incontrolabilidade, a duração do tom, o tipo de feedback e a adição ou subtração de pontos não eram produzidos pelo participante. Entretanto, o participante tinha algum controle sobre esses eventos, uma vez que o responder necessariamente produzia o término do tom e a apresentação do feedback e dos pontos. Esse tipo de controlabilidade poderia facilitar o contato com a controlabilidade em vigor na fase de teste e, assim, atenuar a persistência do responder nessa fase. A segunda característica consiste nos eventos reforçadores e aversivos utilizados. O valor reforçador do feedback de acerto e do ganho de pontos, assim como o valor punitivo do feedback de erro e da perda de pontos, é questionável, uma vez que esses eventos não foram emparelhados com outros estímulos explicitamente reforçadores ou punitivos (e.g., ganho e perda de dinheiro). Conseqüentemente, os efeitos das manipulações experimentais podem ter sido amenizados.

Em resumo, os dados obtidos sugerem que uma história de incontrolabilidade não gera, necessariamente, persistência de padrões comportamentais anteriormente aprendidos (ver também Capelari \& Hunziker, 2005; Sanabio-Heck, 2004). Quando esses padrões produzem reforços na nova contingência, eles tendem a persistir; mas quando não geram reforços, eles são substituídos por padrões mais efetivos. Assim, ao avaliar os efeitos da história de incontrolabilidade, os investigadores devem considerar não somente as variáveis presentes na situação de treino, mas também aquelas presentes nas situações de teste, sendo o desempenho final determinado pela interação entre as situações de treino e teste (Sanabio-Heck \& Motta, 2004). Essa análise é consistente com a argumentação de Skinner (1966) de que o comportamento é determinado por processos seletivos - filogenéticos, ontogenéticos e culturais presentes no ambiente histórico e atual do organismo.

\section{REFERÊNCIAS}

Abreu-Rodrigues, J., Natalino, P. C. \& Aló, R. M. (2002). Instruções e iniqüidade de reforços: efeitos sobre o comportamento competitivo. Psicologia: Teoria $e$ Pesquisa, 18, 83-94.

Capelari, A. \& Hunziker, M. H. L. (2005). Aprendizagem de fuga após estímulos apetitivos incontroláveis. Psicologia: Teoria e Pesquisa, 21, 99-107.

Carvalho, M. V. (1998). Interação entre eventos aversivos controláveis/incontroláveis e reforçamento contínuo parcial: efeitos sobre a sensibilidade à mudança de 
estímulos. Dissertação de Mestrado Não-Publicada. Universidade de Brasília, Brasília.

Danforth, J. S., Chase, P. N., Dolan, M., \& Joyce, J. H. (1990). The establishment of stimulus control by instructions and by differential reinforcement. Journal of the Experimental Analysis of Behavior, 54, 97-112.

Galizio, M. (1979). Contingency-shaped and rule-governed behavior: Instructional control of human loss avoidance. Journal of the Experimental Analysis of Behavior, 31, 5370.

Goodkin, F. (1976). Rats learning the relationship between responding and environmental events: An expansion of the learned helplessness hypothesis. Learning \& Motivation, 7, 382-393.

Hiroto, D. S. (1974). Locus of Control and Learned Helplessness. Journal of Experimental Psychology, 102, 187-193.

Klein, D. C. \& Seligman, M. E. P. (1976). Reversal of performance deficits and perceptual deficits in learned helplessness and depression. Journal of Abnormal Psychology, 85, 11-26.

Klein, D. C., Fencil-Morse, E. \& Seligman, M. E. P. (1976). Learned helplessness, depression and the attribution of failure. Journal of Personality and Social Psychology, 5, 508-516.

Madden, G. J., Chase, P. N. \& Joyce, J. H. (1998). Making sense of sensitivity in the human operant literature. The Behavior Analyst, 21, 1-12.

Mikulincer, M. (1986). Attributional processes in the learned helplessness paradigm: Behavioral effects of global attributions. Journal of Personality and Social Psychology, $51,1248-1256$

Nation, J. R. \& Massad, P. (1978). Persistence training: A partial reinforcement procedure for reversing learned helplessness and depression. Journal of Experimental Psychology: General, 107, 436-451.

Oakes, W. F. \& Curtis, N. (1982). Learned helplessness: Not dependent upon cognitions, attributions, or other such phenomenal experiences. Journal of Personality, 50, 387408.

Rodd, Z. A., Rosellini, R. A., Stock, H. S. \& Gallup, G. G. Jr. (1997). Learned helplessness in chickens (Gallus gallus): Evidence for attentional bias. Learning and Motivation, 28, 43-55.
Sanabio-Heck, E. T. (2004). Efeitos da história de incontrolabilidade e do relato verbal sobre a variação comportamental. Tese de Doutorado Não-Publicada, Programa de Pós-Graduação em Psicologia, Universidade de Brasília, Brasília.

Sanabio-Heck, E. T. \& Motta, K. G. S. (2004). Desamparo aprendido. Em J. Abreu-Rodrigues \& M. R. Ribeiro (Orgs.), Análise do comportamento: pesquisa, teoria $e$ aplicação (pp. 81-98). Porto Alegre: Artmed.

Schoenfeld, W. N. (1968). On the difference in resistance to extinction following regular and periodic reinforcement. Journal of the Experimental Analysis of Behavior, 11, 259261.

Seligman, M. E. P. \& Maier, S. F. (1967). Failure to escape traumatic shock. Journal of Experimental Psychology, 74, $1-9$.

Seligman, M. E. P., Rosellini, R. A. \& Kozak, M. J. (1975). Learned helplessness in the rat: Time course, immunization, and reversibility. Journal of Comparative and Physiological Psychology, 88, 542-547.

Shimoff, E., Matthews, B. A. \& Catania, A. C. (1986). Human operant performance: Sensitivity and pseudosensitivity to contingencies. Journal of the Experimental Analysis of Behavior, 46, 149-157.

Skinner, B. F. (1966). The phylogeny and ontogeny of behavior. Science, 153, 1205-1213.

Tatham, T. A., Wanchisen, B. A. \& Hineline, P. N. (1993). Effects of fixed and variable ratios on human behavioral variability. Journal of the Experimental Analysis of Behavior, 59, 349-359.

Tennen, H., Gillen, R. \& Drum, P. E. (1982). The debilitating effect of exposure to noncontingent escape: A test of the learned helplessness model. Journal of Personality, 50, 409-425.

Williams, J. L. \& Maier, S. F. (1977). Transituational immunization and therapy of learned helplessness in the rat. Journal of Experimental Psychology: Animal Behavior Processes, 3, 240-252.

Winefield, A. H. \& Tiggemann, M. (1978). The effects of uncontrollable unpredictable events on anagram solving. Quarterly Journal of Experimental Pshychology, 30, 717724

Recebido em 21/02/2006 Aceito em 12/02/2007

\section{AGRADECIMENTOS}

As autoras agradecem a Fernando César Alves da Rocha pelo desenvolvimento do software.

Endereço para correspondência: Karina de Guimarães Souto e Motta. SQN 208 Bloco D Apt 106, CEP 70853-040, Brasília-DF. E-mail:karinamotta@terra.com.br 